\title{
Effectiveness of first-line pegvisomant monotherapy in acromegaly: an ACROSTUDY analysis
}

\author{
Nicholas A Tritos', Philippe Chansonn',3, Camilo Jimenez ${ }^{4}$, Donna King ${ }^{5}$, \\ Peter J Jönsson ${ }^{6}$, Anne Klibanski ${ }^{1}$ and Beverly M K Biller ${ }^{1}$
}

${ }^{1}$ Neuroendocrine Unit, Massachusetts General Hospital and Harvard Medical School, Boston, Massachusetts, USA, ${ }^{2}$ Assistance Publique-Hôpitaux de Paris, Hôpitaux Universitaires Paris-Sud, Hôpital de Bicêtre, Service

d'Endocrinologie et des Maladies de la Reproduction, Le Kremlin Bicêtre, France, ${ }^{3}$ Institut National de la Santé et de la Recherche Médicale U1185, Fac Med Paris Sud, Université Paris-Saclay, Le Kremlin-Bicêtre, France, ${ }^{4}$ Endocrine Neoplasia and Hormonal Disorders, The University of Texas MD Anderson Cancer Center, Houston, Texas, USA,

${ }^{5}$ Endocrine Care, Pfizer, Inc, Lawrenceville, New Jersey, USA, and ${ }^{6}$ Pfizer Health AB, Sollentuna, Sweden

Correspondence should be addressed to N A Tritos

Email

ntritos@mgh.harvard.edu

\section{Abstract}

Objective: To examine the effectiveness and safety of primary pegvisomant monotherapy.

Design: Retrospective analysis of data extracted from ACROSTUDY (global observational outcomes study of patients with acromegaly treated with pegvisomant).

Methods: The earliest time to insulin-like growth factor 1 (IGF-1) normalization on pegvisomant monotherapy was determined. Both the proportion of patients who achieved IGF-1 normalization and the time to IGF-1 normalization on pegvisomant monotherapy were assessed.

Results: Eligible patients included 28 subjects on primary medical therapy (PT) and 176 controls on adjunctive pegvisomant therapy treated postoperatively, including 43 who were naïve to medical therapy (NMT) and 133 who were previously treated medically and were washed out (WASH). IGF-1 normalization occurred in 76.9\% (PT), 85.2\% (NMT) and $78.3 \%$ (WASH) patients ( $P=$ NS). Median times to IGF-1 normalization were 0.5 year (PT), 0.7 year (NMT) and 0.6 year $(\mathrm{WASH}), P=\mathrm{NS}$. On survival analysis, the fraction of patients controlled on pegvisomant monotherapy was not different between groups. Higher baseline IGF-1 levels, obtained at study entry, predicted a lower likelihood of IGF-1 normalization on monotherapy $(P=0.012)$. Safety data include low prevalence of skin rashes, injection site reactions and reversible transaminase elevations. There was one patient (NMT) with a verified increase in tumor size. Conclusions: Pegvisomant monotherapy, administered either as primary medical therapy or as adjunctive therapy according to local practice, led to IGF-1 normalization in $>75 \%$ of patients. Pegvisomant monotherapy had a favorable safety profile, consistent with previous observations. Prospective data are needed to further evaluate the role of primary pegvisomant monotherapy in acromegaly.

\section{Introduction}

Transsphenaoidal pituitary surgery (TSS) is the first-line treatment for the majority of patients with acromegaly secondary to a somatotropin-secreting pituitary adenoma (1). Medical therapy has been used adjunctively to control growth hormone (GH) excess in patients who have persistent disease after TSS (1). The effectiveness of
() 2017 European Society of Endocrinology Printed in Great Britain primary medical therapy with a somatostatin receptor agonist (SSA) has been reported in selected groups of patients with acromegaly. This may be particularly appropriate as a treatment option in patients without mass effect, those who are unlikely to be cured by TSS because of tumor involvement of the cavernous sinuses or 
clivus, those with very small tumors that are not evident on imaging studies, and those who are unfit for or who decline surgery (1).

Pegvisomant is a pegylated form of a genetically engineered human GH analog, which acts as a GH receptor antagonist $(2,3)$. Pegvisomant effectively competes with native GH for binding to its cognate receptor without activating downstream GH signaling pathways, thereby preventing GH action and insulin-like growth factor 1 (IGF-1) secretion $(2,3)$. Pegvisomant is efficacious in controlling the effects of GH excess in patients with acromegaly $(4,5)$. In a pivotal clinical trial, pegvisomant therapy resulted in IGF-1 normalization in up to $89 \%$ of patients with acromegaly who were not cured after TSS (4). In published studies, most patients received pegvisomant as adjunctive therapy. Some data have suggested that glycemic control may improve in patients with acromegaly after switching from SSA therapy to pegvisomant (6). Recent guidelines and consensus statements suggest that pegvisomant is an option as adjuvant, but not primary, therapy in patients with acromegaly $(1,7)$. The Endocrine Society guidelines have suggested the use of somatostatin analogs or pegvisomant as options for adjuvant therapy, whereas the consensus statement has suggested the use of somatostatin analogs as a first-line option for adjuvant therapy $(1,7)$. Indeed, the effectiveness and safety of pegvisomant as primary medical therapy in patients who were previously treatment-naive have not been reported.

ACROSTUDY is a global non-interventional safety surveillance study, open to patients with acromegaly who are treated with pegvisomant in routine clinical practice (8). Previously reported data from ACROSTUDY suggested that IGF-1 was normalized in $67.5 \%$ of patients on pegvisomant monotherapy at 5 years (9). In the present study, data from ACROSTUDY were extracted and analyzed in order to characterize the effectiveness and safety of pegvisomant as primary medical therapy in treatmentnaïve patients in comparison with control subjects who received pegvisomant monotherapy postoperatively.

\section{Patients and methods}

All patients were treated by clinicians in individual sites participating in ACROSTUDY. Treatment decisions were based entirely on local practice, and specific information regarding these decisions could not be obtained from the ACROSTUDY database. Written informed consent was obtained from all study subjects at each participating site at the time of enrollment.
The study was conducted according to the principles of the Declaration of Helsinki (10).

All data were extracted from ACROSTUDY records, including information on age at diagnosis of acromegaly, chronologic age, gender, body mass index (BMI), tumor size (dichotomously reported as microadenoma or macroadenoma), serum IGF-1 level (measured locally and expressed as 'fold elevation' above the upper end of the normal range, based on institutionally-derived normative data for each assay), diabetes mellitus (DM), hypertension, concurrently administered medications, the presence of additional pituitary hormone deficiencies, pegvisomant dose, administration frequency and treatment duration, as well as information on therapies for acromegaly administered either before or after the pegvisomant monotherapy period (TSS, radiation therapy, medical therapies). The diagnosis of DM was made according to the American Diabetes Association guidelines, including fasting plasma glucose $\geq 126 \mathrm{mg} / \mathrm{dL}$, random plasma glucose $\geq 200 \mathrm{mg} / \mathrm{dL}$ or glycosylated hemoglobin A1c (HbA1c) $\geq 6.5 \%$ (11).

The ACROSTUDY database, containing data on 2043 patients (enrolled from database inception through November 2014), was searched for patients meeting the following inclusion criteria: age $\geq 18$ years at study entry, diagnosis of acromegaly or gigantism, being treatmentnaïve at enrollment and receiving pegvisomant as primary medical monotherapy (PT). Two control groups were chosen, including patients receiving adjunctive pegvisomant monotherapy after TSS, who were either naïve to medical therapy (NMT) or previously treated medically with other agents and washed out (WASH).

Patients were excluded if they met any of the following criteria: medical therapy with an SSA or dopamine agonist (DA) within 4 months from study entry or history of previous radiation therapy to the sella. Patients whose baseline (at study entry) serum IGF-1 values were missing, including 15 PT, 16 NMT and $50 \mathrm{WASH}$, were included in safety analyses, but were excluded from the analysis of the effectiveness of pegvisomant.

The primary effectiveness endpoint was defined as the earliest time at IGF-1 normalization on pegvisomant monotherapy. Both the proportion of patients who achieved IGF-1 normalization and the time to IGF-1 normalization on pegvisomant monotherapy were determined.

The Chi square or the Fisher's exact test was used to analyze differences between proportions (as appropriate). Either the Wilcoxon rank sum test or the $t$-test was used to analyze differences between continuous variables (as appropriate). Global testing followed by pairwise tests was used as needed. 
The Kaplan-Meier (product limit) method was used to estimate the fraction of patients who were controlled on pegvisomant monotherapy. In this analysis, patients were followed to the date of the first IGF-1 normalization on pegvisomant monotherapy (event) or, if the event did not occur, patients' data were censored at the time of the last reported non-normal IGF-1 on pegvisomant monotherapy.

Multivariate logistic regression analyses were used to identify predictors of biochemical response, using serum IGF-1 level (dichotomously reported as either normal or not) as the dependent variable. Independent variables introduced in logistic regression analyses include treatment group, age, gender, BMI, baseline IGF-1 level (obtained at study entry), tumor size (dichotomously reported as microadenoma or macroadenoma), prevalent $\mathrm{DM}$, insulin use, presence of additional pituitary hormone deficiencies, pegvisomant dose, administration frequency (daily vs less frequently than daily) and treatment duration.

Statistical analyses were conducted using the Statistical Analysis System (SAS, Institute, Inc, Cary, NC, USA). Data are presented as median (10th percentile, 90th percentile) or percentages. $P$ values $<0.05$ were considered statistically significant.

\section{Results}

\section{Patient demographics and comorbidities}

Database searches identified 28 PT, 43 NMT and 133 WASH patients who were eligible for inclusion in the present study out of 2043 subjects within ACROSTUDY. Study subjects originated from 97 ACROSTUDY sites out of a total of 304 participating centers. Baseline demographic and clinical characteristics of the study population are shown in Table 1 . The vast majority of patients in the study had GH excess of adult onset; only one patient in the WASH group had gigantism. There was no difference in age or gender distribution, prevalent DM, hypertension, liver disease, or median pegvisomant dose at study entry between the PT, NMT and WASH groups. There were 59 patients with DM, including one patient in the NMT group who had type $1 \mathrm{DM} ; 18$ patients (2 PT, 5 NMT and 11 WASH) had type 2 DM. The type of DM was not defined in 40 patients (6 PT, 7 NMT and 27 WASH). Median HbA1c levels were $6.5 \%(6.1,6.9)$ in PT, $7.3 \%$ $(6.3,8.3)$ in NMT, and $5.9 \%(5.0,7.8)$ in WASH $(P=0.045$, between NMT vs WASH; differences between PT vs NMT, and PT vs WASH were not significant). No patients were taking oral estrogens. Patients in the PT group had a higher median BMI than those in WASH $(P=0.02)$.

\section{Tumor characteristics and IGF-1 values at baseline (study entry)}

The proportion of patients with microadenoma was significantly higher in the PT group in comparison with the two control groups $(P<0.03$, Table 1$)$. At study entry, the presence of a visible lesion, consistent with adenoma on pituitary imaging, was reported in 24 (85.7\%) PT, 32 (74.4\%) NMT, and 90 (67.7\%) WASH; lateral tumor extension (in the cavernous sinus) was reported in

Table 1 Baseline demographic and clinical characteristics of the study population.

\begin{tabular}{|c|c|}
\hline & PT $(n=28)$ \\
\hline Age (year)* & $48.5(22.2,67.6)$ \\
\hline Gender (F/M (\%/\%)) & $14 / 14(50 / 50)$ \\
\hline BMI $\left(\mathrm{kg} / \mathrm{m}^{2}\right)^{*}$ & $33.5(27.4,41.3)$ \\
\hline Diabetes mellitus (\%) & $8(28.6)$ \\
\hline Hypertension (\%) & $15(53.6)$ \\
\hline Liver disease (\%) & $0(0)$ \\
\hline Microadenoma (\%) & $8(28.6)$ \\
\hline IGF-1 (fold ULN)* & $2.9(1.2,4.8)$ \\
\hline $\begin{array}{l}\text { Interval between diagnosis to start } \\
\text { pegvisomant (year)* }\end{array}$ & $0.2(0,3.1)$ \\
\hline $\begin{array}{l}\text { Pegvisomant starting dose at } \\
\text { study entry (mg/day)* }\end{array}$ & $10(10,30)$ \\
\hline
\end{tabular}

\begin{tabular}{c}
\hline NMT $(n=43)$ \\
\hline $48.7(27.8,64.9)$ \\
$20 / 23(46.5 / 53.5)$ \\
$29.4(24.9,47.2)$ \\
$13(30.2)$ \\
$17(39.5)$ \\
$3(6.9)$ \\
$\mathbf{3}(6.9)$ \\
$\mathbf{1 . 9}(1.1,3.7)$ \\
$\mathbf{1 . 0}(0.3,13.4)$ \\
$10(10,20)$
\end{tabular}

\begin{tabular}{c}
\hline WASH $(n=133)$ \\
\hline $51.3(29.9,66.1)$ \\
$69 / 64(51.9 / 48.1)$ \\
$28.7(23.2,36.2)$ \\
$38(28.6)$ \\
$61(45.9)$ \\
$16(12.0)$ \\
$14(10.7)$ \\
$1.6(0.8,3.2)$ \\
$\mathbf{4 . 7}(1.2,17.8)$
\end{tabular}

$10(10,20)$

\begin{tabular}{c}
\hline $\boldsymbol{P}$ Value \\
\hline NS \\
NS \\
$\mathbf{0 . 0 2}$ \\
NS \\
NS \\
NS \\
$<\mathbf{0 . 0 3} \mathbf{3}^{* *}$ \\
$<\mathbf{0 . 0 5}$ \\
$<\mathbf{0 . 0 0 1}^{\dagger \dagger}$
\end{tabular}

NS

All differences between values shown in bold are statistically significant both on global and pairwise statistical testing (values not shown in bold are not significantly different).

${ }^{*}$ Continuous data represent median (10th percentile, 90th percentile). **Pairwise comparisons between PT vs NMT; PT vs WASH. ${ }^{\dagger}$ Pairwise comparisons between PT vs WASH; NMT vs WASH. ${ }^{\dagger}$ Pairwise comparisons between PT vs NMT; PT vs WASH; NMT vs WASH.

BMI, body mass index; IGF-1, insulin-like growth factor 1; NMT, naive to medical treatment; NS, not significant; PT, primary medical monotherapy; ULN, upper limit of normal; WASH, previously medically treated and washed out. 
Table 2 Data on IGF-1 normalization on pegvisomant monotherapy.*

\begin{tabular}{|c|c|c|c|c|}
\hline & PT $(n=13)$ & NMT $(n=27)$ & WASH $(n=83)$ & $P$ Value \\
\hline Patients who achieved normal IGF-1 n (\%) & $10(76.9)$ & $23(85.2)$ & $65(78.3)$ & NS \\
\hline Time to IGF-1 normalization (year)† & $0.5(0.2,2.5)$ & $0.7(0.1,2.3)$ & $0.6(0.1,2.5)$ & NS \\
\hline Pegvisomant dose $(\mathrm{mg} / \mathrm{day})^{\dagger}$ & $15(10,30)$ & $10(10,20)$ & $10(9,19)$ & 0.03 \\
\hline
\end{tabular}

All differences between values shown in bold are statistically significant both on global and pairwise statistical testing (values not shown in bold are not significantly different).

*Analysis restricted to patients with elevated baseline IGF-1 levels. ${ }^{\dagger}$ Continuous data represent median (10th percentile, 90th percentile). For each patient, the mean pegvisomant dose received during monotherapy was calculated. Mean pegvisomant doses were as follows: $17 \mathrm{mg} / \mathrm{day}$ (PT group); $13 \mathrm{mg} /$ day (NMT group); $12 \mathrm{mg} /$ day (WASH group).

IGF-1, insulin-like growth factor 1; NMT, naive to medical treatment; NS, not significant; PT, primary medical monotherapy; WASH, previously medically treated and washed out.

6 (21.4\%) PT, 17 (39.5\%) NMT, and 39 (29.3\%) WASH; suprasellar extension was reported in 6 (21.4\%) PT, 13 (30.2\%) NMT, and 17 (12.8\%) WASH; inferior extension (in the sphenoid or sphenoid sinus) was reported in 2 (7.1\%) PT, 2 (4.6\%) NMT and 8 (6.0\%) WASH. At baseline, patients in the PT group had a higher median IGF-1 level in comparison with WASH, and those in the NMT group had a higher median IGF-1 level than WASH $(P<0.05)$. The interval between the diagnosis of acromegaly and the onset of pegvisomant monotherapy was shorter in the PT group in comparison with the two control groups $(P<0.001)$.

\section{Effectiveness}

Similar proportions of patients in the PT (76.9\%), NMT (85.2\%) and WASH (78.3\%) groups achieved IGF-1 normalization ( $P=\mathrm{NS}$, Table 2 ). The median time to IGF-1 normalization was not different between the three groups $(P=$ NS, Table 2$)$. Pegvisomant doses during monotherapy were higher in the PT group in comparison with WASH $(P=0.03$, Table 2$)$. Stratified by baseline IGF-1 level (expressed as 'fold' elevation above the upper limit of normal (ULN) as follows: $\leq 2,2.1-3$, 3.1-4 and $>4$ ), the proportion of patients (expressed as percentage) whose IGF-1 normalized on pegvisomant monotherapy was $0,100,50,75$ (PT); 80, 100, 100, 66.7 (NMT); and 86.7, 75, 72.7, 0 (WASH).

On Kaplan-Meier analysis, the proportion of patients who were controlled on pegvisomant monotherapy did not differ between PT, NMT and WASH ( $P=$ NS, Fig. 1$)$. There was no significant change (from baseline) in HbA1c levels (examined as measure of glycemic control) during pegvisomant monotherapy among patients with DM in each of the 3 groups (PT, NMT or WASH; data not shown).

The median time on pegvisomant monotherapy was shorter in PT in comparison with WASH, or in
NMT in comparison with WASH $(P<0.02$, Table 3$)$. The proportion of patients in the PT group who underwent TSS after pegvisomant monotherapy $(35.7 \%)$ was higher than in the NMT $(6.9 \%)$ or WASH $(4.5 \%)$ groups $(P<0.005$, Table 3$)$. In contrast, neither the proportion of patients who underwent radiation therapy nor the proportion of patients who received add-on SSA or DA therapy after pegvisomant monotherapy was different between PT, NMT and WASH groups ( $P=$ NS, Table 3$)$. Recommendations for radiation therapy were made at the discretion of the treating physicians, who did not record their rationale in ACROSTUDY. Pegvisomant therapy was restarted after administration of radiation therapy in 33 out of 40 patients, including 7 in PT, 9 in NMT and 17 in WASH group, respectively. At the time of the last visit, similar proportions of patients in the PT, NMT and WASH groups remained on pegvisomant monotherapy $(P=$ NS, Table 3$)$.

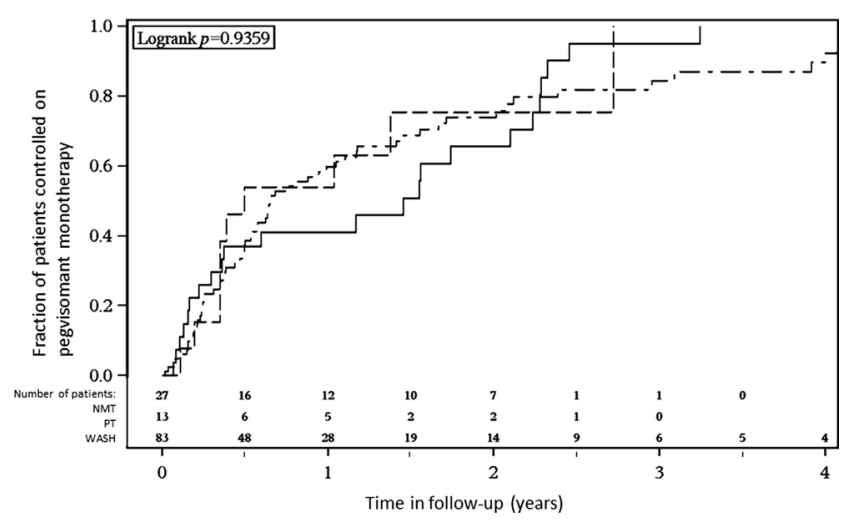

Figure 1

Fraction of patients controlled on pegvisomant monotherapy. NMT, naive to medical treatment (continuous line); PT, primary medical monotherapy (dashed line); WASH, previously medically treated and washed out (dotted/dashed line). 
Table 3 Data on patient follow-up and subsequent therapeutic interventions at the end of the pegvisomant monotherapy period.

\begin{tabular}{l}
\hline \\
\hline Time on pegvisomant monotherapy (year)* \\
Patients who underwent pituitary surgery after \\
pegvisomant monotherapy $(\%)$ \\
Patients who underwent radiation therapy after \\
pegvisomant monotherapy (\%) \\
Patients who added SSA or DA (\%) \\
Patients remaining on pegvisomant monotherapy at \\
the last visit $(\%)$
\end{tabular}

\begin{tabular}{c}
\hline $\mathbf{P T}(n=28)$ \\
\hline $\mathbf{1 . 6}(0.5,6.5)$ \\
$\mathbf{1 0}(\mathbf{3 5 . 7})$ \\
$7(25.0)$ \\
$11(39.3)$ \\
$12(42.9)$ \\
\hline
\end{tabular}

\begin{tabular}{c}
\hline NMT $(n=43)$ \\
\hline $\mathbf{2 . 9}(0.3,6.9)$ \\
$\mathbf{3}(6.9)$ \\
$10(23.3)$ \\
$17(39.5)$ \\
$20(46.5)$
\end{tabular}

\begin{tabular}{|c|}
\hline WASH $(n=133)$ \\
\hline $\begin{array}{c}3.8(0.6,8.4) \\
6(4.5)\end{array}$ \\
\hline $23(17.3)$ \\
\hline $\begin{array}{l}38(28.6) \\
78(58.6)\end{array}$ \\
\hline
\end{tabular}

\begin{tabular}{c}
\hline P Value \\
\hline$<\mathbf{0 . 0 2}{ }^{\dagger}$ \\
$<\mathbf{0 . 0 0 5}^{\dagger \dagger}$
\end{tabular}

All differences between values shown in bold are statistically significant both on global and pairwise statistical testing (values not shown in bold are not significantly different).

*Continuous data represent median (10th percentile, 90th percentile). ${ }^{\dagger}$ Comparisons between PT vs WASH; NMT vs WASH. ${ }^{+\dagger}$ Comparisons between PT vs NMT; PT vs WASH.

DA, dopamine agonist; IGF-1, insulin-like growth factor 1; NMT, naive to medical treatment; NS, not significant; PT, primary medical monotherapy; SSA, somatostatin analog; WASH, previously medically treated and washed out.

Multivariate logistic regression analyses were conducted to identify the potential predictors of biochemical response (as detailed under 'Patients and methods' section). In the entire study population, a higher baseline (at study entry) IGF-1 level predicted a lower likelihood of IGF-1 normalization during pegvisomant monotherapy (odds ratio (OR) and 95\% confidence interval $(\mathrm{CI})=0.62(0.42,0.90), P=0.012)$. In the WASH group, a higher baseline IGF-1 level predicted a lower likelihood of IGF-1 normalization during pegvisomant monotherapy $(\mathrm{OR}=0.31(0.15,0.67), P=0.003)$. In addition, in the WASH group, prevalent $\mathrm{DM}$ at study entry predicted a lower likelihood of IGF-1 normalization, albeit with marginal statistical significance $(\mathrm{OR}=0.26(0.07,1.00), P=0.051)$. No predictors of IGF-1 normalization were identified when data on PT and NMT groups were separately analyzed, likely because of their small sample size.

\section{Safety data}

Pegvisomant was temporarily or permanently withdrawn in 3 patients in PT, 5 in NMT and 10 in WASH (Table 4). Reported reasons for pegvisomant withdrawal include transaminase elevations in 3, injection site reactions/skin rashes in 2, lack of therapeutic response in 2, cholecystitis in 1 , headache in 1 , renal failure in 1 , hypophagia in 1 , maternal exposure during pregnancy in 1 , and benign lung mass in 1 patient (Table 4). Reversible abnormalities in liver chemistries and injection site reactions were reported in a small number of patients (Table 4).

During the pegvisomant monotherapy period, data on follow-up magnetic resonance imaging (MRI) of the sella were available in 23/28 patients (82.1\%) in PT, 31/43 $(72.1 \%)$ in NMT, and $101 / 133(75.9 \%)$ in WASH $(P=\mathrm{NS}$ between groups). Locally interpreted magnetic resonance imaging (MRI) examinations of the sella were interpreted to show evidence of tumor growth in 2 patients in PT, 5 patients in NMT and 9 patients in WASH. Of these 16 cases, which were all reanalyzed centrally, there was only 1 patient (in the NMT group) with verified tumor growth during pegvisomant monotherapy (Table 4). The increase in tumor size was not thought to be clinically significant in this patient, who continued on pegvisomant monotherapy. No further increase in tumor size was reported on subsequent pituitary imaging, obtained 12 months and 28 months later.

Table 4 Safety data on pegvisomant monotherapy.

\begin{tabular}{|c|c|c|c|}
\hline & PT $(n=28)$ & NMT $(n=43)$ & WASH $(n=133)$ \\
\hline $\begin{array}{l}\text { Pegvisomant withdrawal (temporary or } \\
\text { permanent }(\%))^{*}\end{array}$ & $3(10.7)$ & $5(11.6)$ & $10(7.5)$ \\
\hline Abnormal liver chemistries (\%) & $1(3.6)$ & $1(2.3)$ & $4(3.0)$ \\
\hline Injection site reactions (\%) & $1(3.6)$ & $1(2.3)$ & $2(1.5)$ \\
\hline Increase in tumor size (centrally verified (\%)) & $0(0)$ & $1(2.3)$ & $0(0)$ \\
\hline
\end{tabular}

*Reasons for pegvisomant withdrawal (n): transaminase elevations (3), injection site reactions/skin rashes (2), lack of therapeutic response (2), cholecystitis (1), headache (1), renal failure (1), hypophagia (1), maternal exposure during pregnancy (1) and (benign) lung mass (1). NMT, naive to medical treatment; PT, primary medical monotherapy; WASH, previously medically treated and washed out. 


\section{Discussion}

Pegvisomant is effective in controlling IGF-1 secretion in the majority of patients with acromegaly who are treated postoperatively, either alone (as monotherapy) or in combination with an SSA or DA $(1,12,13,14,15)$. These data have led to regulatory approval of pegvisomant (in the USA, Europe and other countries) as adjunctive therapy in patients not cured by surgery. However, the effectiveness of pegvisomant monotherapy as primary medical therapy in acromegaly has not been systematically examined. Recent guidelines and consensus statements do not include pegvisomant as a primary therapy option in treatment-naïve patients with acromegaly $(1,7)$. These observations highlight the novelty of the present study.

In the present retrospective study, observational data from ACROSTUDY were extracted and analyzed in order to evaluate the effectiveness of pegvisomant as primary monotherapy in treatment-naive patients (PT) in comparison with those on pegvisomant monotherapy treated postoperatively (NMT and WASH groups). Data from the present study suggest that pegvisomant monotherapy was effective in normalizing serum IGF-1 levels in over $75 \%$ of treated patients in each of the 3 groups (PT, NMT and WASH) before any additional treatment was administered (TSS, radiation therapy and other medications). The fraction of patients who normalized their IGF-1 levels on pegvisomant monotherapy was not different between those on primary pegvisomant monotherapy and those in the 2 comparator groups (NMT and WASH). These data suggest that primary pegvisomant monotherapy was effective in achieving biochemical control of excess GH action in the majority of carefully selected (by their treating physicians) groups of patients with acromegaly regardless of whether they were treated before or after TSS. The effectiveness of pegvisomant appears to be higher than previously reported in the 'real world' (IGF-1 normalization in $67.5 \%$ of patients within ACROSTUDY at a mean dose of $17.2 \mathrm{mg} /$ day), but lower than that reported in a clinical trial (IGF-1 normalization in $89 \%$ of patients at a dose of $20 \mathrm{mg} /$ day) $(4,9)$. It is possible that differences in population characteristics or variable adherence to therapy might account for these differences in effectiveness. However, this remains a matter of speculation.

All decisions on therapy of patients in the present study, including those in the primary pegvisomant monotherapy (PT group), were made by individual site investigators. It must be emphasized that the present report reflects pegvisomant use according to local practice. Therefore, it is not possible to ascertain why primary medical therapy with pegvisomant (rather than a somatostatin analog) was chosen. Patients in the PT group were more likely to have a pituitary microadenoma and had a higher median IGF-1 level at baseline (study entry) in comparison with the control population, suggesting that their physicians may have considered tumor size and biochemical severity of acromegaly in making treatment decisions.

There was no evident improvement in glycemic control (based on HbA1c levels) in the subgroups of PT, NMT or WASH patients with DM who received pegvisomant therapy, in contrast to previously published data $(6,16)$. However, meaningful interpretation of these findings is hampered by the small size of these subgroups. It is also not known if glycemic control improved in patients in the WASH group after switching from SSA to pegvisomant (at ACROSTUDY entry). After undergoing primary pegvisomant monotherapy, $35.7 \%$ of patients in the PT group underwent pituitary surgery (TSS) as definitive therapy. The role of pegvisomant monotherapy as preoperative therapy, aimed at ameliorating some of the acromegaly-related comorbidities (including DM, upper airway narrowing and sleep apnea), requires further study, given available data on the efficacy of SSA administered preoperatively (17).

Regardless of whether pegvisomant therapy was primary (PT group) or adjunctive (NMT and WASH groups), a higher IGF-1 level at study entry predicted a lower likelihood of IGF-1 normalization on pegvisomant monotherapy, in agreement with previously published data $(18,19)$. This finding is predicated by the mechanism of action of pegvisomant, which involves drug competition with endogenous native $\mathrm{GH}$ for its cognate receptor. In contrast, neither pegvisomant dose nor frequency of administration was a predictor of biochemical response.

Of note, the pegvisomant dose range prescribed by clinicians in the present study was fairly narrow. The product label approved in the USA, Canada and the European Union countries specifies a pegvisomant dose between $10 \mathrm{mg}$ and $30 \mathrm{mg}$ daily. It is conceivable that pegvisomant dose uptitration was not sufficient to normalize IGF-1 levels in some patients in the study, possibly as a consequence of lack of availability of higher pegvisomant dose vials (containing $>20 \mathrm{mg} / \mathrm{vial}$ ) in the past. Other potential explanations include lack of appreciation of the need to uptitrate pegvisomant dose or the cost of higher pegvisomant doses. Indeed, most patients (90\%) in the NMT and WASH groups did not receive pegvisomant doses higher than $20 \mathrm{mg}$ daily, 
suggesting that dose uptitration may have not been sufficient in some patients.

In the WASH group, prevalent DM (at study entry) was associated with a lower likelihood of IGF-1 normalization on pegvisomant monotherapy (with borderline statistical significance), in agreement with previously published data (20). This intriguing finding may reflect an effect of insulin on $\mathrm{GH}$ receptor expression and turnover in hepatocytes, which are the source of the majority of systemic IGF-1. Insulin has been reported to modulate $\mathrm{GH}$ receptor expression and turnover in a hepatoma cell line (21). It is therefore possible that some patients with DM may be less responsive to pegvisomant effect as a consequence of expressing a higher number of $\mathrm{GH}$ receptors on hepatocytes than non-diabetic patients. However, further data are needed to confirm this postulated mechanism.

In the present study, pegvisomant monotherapy was associated with a low prevalence of adverse effects, including local injection reactions, skin rashes, reversible transaminase elevations, and a low risk of pituitary tumor progression (1/204 patients in the present report). In a previous study, tumor growth was reported in 2 out of 160 patients $(1.2 \%)$ who received pegvisomant for up to 18 months (5). In a large report from ACROSTUDY, centrally verified growth of pituitary adenomas was reported in $3.2 \%$ out of 936 patients who had at least 2 MRI examinations available for review (22). The present data are in broad agreement with previous observations. These data affirm previous reports on the safety of pegvisomant therapy, which have been overall reassuring $(22,23)$. In agreement with previous publications, pegvisomant therapy does not appear to predispose to tumor progression (22). Nevertheless, adenoma growth may occur in a minority of treated patients (22). In some studies, tumor progression has been reported in pegvisomant-treated patients with aggressive tumors or after withdrawal of SSA therapy, so it is unclear if this is attributable to pegvisomant $(22,24)$. Since pegvisomant lacks direct tumor antiproliferative effect by virtue of its mechanism of action, it is advisable to obtain regular pituitary imaging in patients receiving monotherapy.

The current study has some limitations arising from its retrospective design and lack of availability of some data in ACROSTUDY. Since baseline GH levels were not available in most patients, it was not possible to examine whether GH levels, measured at baseline, may predict IGF-1 normalization on pegvisomant monotherapy. No information is available as to the reasons patients were washed out of other medications. In addition, specific information regarding treatment decisions could not be obtained from ACROSTUDY. The present study represents a report of clinical experience in a select patient population and is not a clinical trial. However, the findings of the present study are intriguing and suggest that the role of primary pegvisomant monotherapy needs further evaluation in a prospective study, including patients treated preoperatively with an aim to potentially ameliorate acromegaly-related comorbidities, improve glycemic control, and mitigate airway management problems that may occur in patients with acromegaly during surgery.

In conclusion, pegvisomant monotherapy, administered either as primary medical therapy or as adjunctive (postoperative) therapy according to local practice, led to IGF-1 normalization in over $75 \%$ of treated patients. Higher baseline IGF-1 levels and prevalent DM were predictive of lower likelihood of IGF-1 normalization on pegvisomant monotherapy in the dose range reported herein; dose uptitration may have been inadequate. Pegvisomant monotherapy was overall welltolerated with a favorable safety profile, in agreement with previous observations. Prospective data are needed in order to better define the role of primary pegvisomant monotherapy in patients with acromegaly.

\section{Declaration of interest}

ACROSTUDY is sponsored by Pfizer, Inc. N A T, P C, C J, A K and B M K B were not compensated for their participation in the present study. N A T has received institution-directed research support from Ipsen, Novartis, Novo Nordisk and Pfizer, Inc; P C has received institution-directed research and educational support from Ipsen, Novartis, Antisense and Pfizer, Inc; C J has no conflicts of interest to disclose; D K and P J J are employees of Pfizer, Inc; A K has no conflicts of interest to disclose; B M K B has received institution-directed research support from Novartis and consulting honoraria from Ipsen, Novartis and Pfizer, Inc.

\section{Funding}

This research did not receive any specific grant from any funding agency in the public, commercial or not-for-profit sector.

\section{Acknowledgments}

The authors thank all ACROSTUDY investigators, physicians, nurses, staff and patients for their participation.

\section{References}

1 Katznelson L, Laws ER Jr, Melmed S, Molitch ME, Murad MH, Utz A, Wass JA \& Endocrine S. Acromegaly: an endocrine society clinical practice guideline. Journal of Clinical Endocrinology and Metabolism 201499 3933-3951. (doi:10.1210/jc.2014-2700)

2 Kopchick JJ. Discovery and mechanism of action of pegvisomant. European Journal of Endocrinology 2003148 (Supplement 2) S21-S25. (doi:10.1530/eje.0.148S021) 
3 Maamra M, Kopchick JJ, Strasburger CJ \& Ross RJ. Pegvisomant, a growth hormone-specific antagonist, undergoes cellular internalization. Journal of Clinical Endocrinology and Metabolism 2004 89 4532-4537. (doi:10.1210/jc.2003-031781)

4 Trainer PJ, Drake WM, Katznelson L, Freda PU, Herman-Bonert V, van der Lely AJ, Dimaraki EV, Stewart PM, Friend KE, Vance ML et al. Treatment of acromegaly with the growth hormone-receptor antagonist pegvisomant. New England Journal of Medicine $20003 \mathbf{3 4 2}$ 1171-1177. (doi:10.1056/NEJM200004203421604)

5 van der Lely AJ, Hutson RK, Trainer PJ, Besser GM, Barkan AL, Katznelson L, Klibanski A, Herman-Bonert V, Melmed S, Vance ML et al. Long-term treatment of acromegaly with pegvisomant, a growth hormone receptor antagonist. Lancet 2001 358 1754-1759. (doi:10.1016/s0140-6736(01)06844-1)

6 Barkan AL, Burman P, Clemmons DR, Drake WM, Gagel RF, Harris PE, Trainer PJ, van der Lely AJ \& Vance ML. Glucose homeostasis and safety in patients with acromegaly converted from long-acting octreotide to pegvisomant. Journal of Clinical Endocrinology and Metabolism 200590 5684-5691. (doi:10.1210/jc.2005-0331)

7 Giustina A, Chanson P, Kleinberg D, Bronstein MD, Clemmons DR, Klibanski A, van der Lely AJ, Strasburger CJ, Lamberts SW, Ho KK et al. Expert consensus document: a consensus on the medical treatment of acromegaly. Nature Reviews Endocrinology 201410 243-248. (doi:10.1038/nrendo.2014.21)

8 Trainer PJ. ACROSTUDY: an overview. Hormone Research 200768 (Supplement 5) 68-69. (doi:10.1159/000110480)

9 Freda PU, Gordon MB, Kelepouris N, Jonsson P, KoltowskaHaggstrom M \& van der Lely AJ. Long-term treatment with pegvisomant as monotherapy in patients with acromegaly: experience from acrostudy. Endocrine Practices 201521 264-274. (doi:10.4158/ EP14330.OR)

10 Riis P. Thirty years of bioethics: the Helsinki Declaration 1964-2003. New Review of Bioethics 20031 15-25. (doi:10.1080/174002803200013 1396)

11 American Diabetes Association. Diagnosis and classification of diabetes mellitus. Diabetes Care 201033 (Supplement 1) S62-S69. (doi:10.2337/dc10-S062)

12 Buchfelder M, Schlaffer S, Droste M, Mann K, Saller B, Brubach K, Stalla GK, Strasburger CJ \& German Pegvisomant Observational Study. The German ACROSTUDY: past and present. European Journal of Endocrinology 2009161 (Supplement 1) S3-S10. (doi:10.1530/EJE09-0350)

13 Chanson P, Brue T, Delemer B, Caron P, Borson-Chazot F, Zouater H \& Medecins de l'Etude ACROSTUDY. Pegvisomant treatment in patients with acromegaly in clinical practice: the French ACROSTUDY. Annales D'Endocrinologie 201576 664-670. (doi:10.1016/j.ando.2015.10.003)

14 Muhammad A, Van der Lely AJ, O'Connor RD, Delhanty PJ, Dal J, Dallenga AH, Feelders RA, Janssen JA, Jorgensen JO \& Neggers SJ. What is the efficacy of switching to weekly pegvisomant in acromegaly patients well controlled on combination therapy?
European Journal of Endocrinology 2016174 663-667. (doi:10.1530/EJE15-1150)

15 Neggers SJ, van Aken MO, Janssen JA, Feelders RA, de Herder WW $\&$ van der Lely AJ. Long-term efficacy and safety of combined treatment of somatostatin analogs and pegvisomant in acromegaly. Journal of Clinical Endocrinology and Metabolism 200792 4598-4601. (doi:10.1210/jc.2007-1234)

16 Drake WM, Rowles SV, Roberts ME, Fode FK, Besser GM, Monson JP $\&$ Trainer PJ. Insulin sensitivity and glucose tolerance improve in patients with acromegaly converted from depot octreotide to pegvisomant. European Journal of Endocrinology 2003149 521-527. (doi:10.1530/eje.0.1490521)

17 Jacob JJ \& Bevan JS. Should all patients with acromegaly receive somatostatin analogue therapy before surgery and, if so, for how long? Clinical Endocrinology 201481 812-817. (doi:10.1111/ cen.12553)

18 Parkinson C, Burman P, Messig M \& Trainer PJ. Gender, body weight, disease activity, and previous radiotherapy influence the response to pegvisomant. Journal of Clinical Endocrinology and Metabolism 200792 190-195. (doi:10.1210/jc.2006-1412)

19 Sievers C, Baur DM, Schwanke A, Buchfelder M, Droste M, Mann K $\&$ Stalla GK. Prediction of therapy response in acromegalic patients under pegvisomant therapy within the German ACROSTUDY cohort. Pituitary 201518 916-923. (doi:10.1007/s11102-015-0673-2)

20 Droste M, Domberg J, Buchfelder M, Mann K, Schwanke A, Stalla G \& Strasburger CJ. Therapy of acromegalic patients exacerbated by concomitant type 2 diabetes requires higher pegvisomant doses to normalise IGF1 levels. European Journal of Endocrinology 2014171 59-68. (doi:10.1530/EJE-13-0438)

21 Leung KC, Doyle N, Ballesteros M, Waters MJ \& Ho KK. Insulin regulation of human hepatic growth hormone receptors: divergent effects on biosynthesis and surface translocation. Journal of Clinical Endocrinology and Metabolism 200085 4712-4720. (doi:10.1210/ jcem.85.12.7017)

22 van der Lely AJ, Biller BM, Brue T, Buchfelder M, Ghigo E, Gomez R, Hey-Hadavi J, Lundgren F, Rajicic N, Strasburger CJ et al. Long-term safety of pegvisomant in patients with acromegaly: comprehensive review of 1288 subjects in ACROSTUDY. Journal of Clinical Endocrinology and Metabolism 201297 1589-1597. (doi:10.1210/ jc.2011-2508)

23 Bernabeu I, Pico A, Venegas E, Aller J, Alvarez-Escola C, GarciaArnes JA, Marazuela M, Jonsson P, Mir N, Garcia Vargas M et al. Safety of long-term treatment with Pegvisomant: analysis of Spanish patients included in global ACROSTUDY. Pituitary 201619 127-137. (doi:10.1007/s11102-015-0691-0)

24 Jimenez C, Burman P, Abs R, Clemmons DR, Drake WM, Hutson KR, Messig M, Thorner MO, Trainer PJ \& Gagel RF. Follow-up of pituitary tumor volume in patients with acromegaly treated with pegvisomant in clinical trials. European Journal of Endocrinology 2008159 517-523. (doi:10.1530/EJE-08-0205)

Received 16 August 2016

Revised version received 4 November 2016

Accepted 15 November 2016 